\title{
Microarray data re-annotation reveals specific IncRNAs and their potential functions in non-small cell lung cancer subtypes
}

\author{
DONGBO ZHOU, MINGXUAN XIE, BAIMEI HE, YING GAO, QIAO YU, BIXIU HE and QIONG CHEN \\ Department of Gerontology, Xiangya Hospital of Central South University, \\ Changsha, Hunan 410078, P.R. China
}

Received April 22, 2016; Accepted April 24, 2017

DOI: $10.3892 / \mathrm{mmr} .2017 .7244$

\begin{abstract}
Non-small-cell lung cancer (NSCLC) is a leading cause of cancer mortality worldwide. The most common subtypes of NSCLC are adenocarcinoma (AC) and squamous cell carcinoma (SCC). However, the pathophysiological mechanisms contributing to AC and SCC are still largely unknown, especially the roles of long non-coding RNAs (lncRNAs). The present study identified differentially expressed lncRNAs between lung AC and SCC by re-annotation of NSCLC microarray data analysis profiling. The potential functions of lncRNAs were predicted by using coding-non-coding gene co-expressing network. Reverse transcription-quantitative polymerase chain reaction (RT-qPCR) was used to investigate lncRNA expression levels in AC cell lines (A549 and L78), SCC cell lines (H226 and H520) and normal cells (NL-20). Western blotting analysis was used to investigate the protein expression levels in these cell lines. A total of 65 IncRNAs were differentially expressed between AC and SCC including $28 \mathrm{lncRNAs}$ that were downregulated in SCC subtypes compared with those in AC ones, and 37 upregulated lncRNAs in SCC subtypes compared with AC subtypes. Three lncRNAs, sex determining region Y-box 2 overlapping transcript (SOX2-OT), NCBP2 antisense RNA 2 (NCBP2-AS2) and ubiquitin like with PHD and ring finger domains 1 (UHRF1), were predicted to be associated with lung cancer; RT-qPCR confirmed that SOX2-OT and NCBP2-AS2 were associated with lung cancer. Finally, western blot assays demonstrated that there was no difference in $\beta$-catenin and glycogen synthase kinase $3 \beta$ (GSK-3 $\beta$ ) expression in cancer cells compared with NL-20, but increased phosphorylated $(\mathrm{p}-) \beta$-catenin and $\mathrm{p}-\mathrm{GSK}-3 \beta$ was detected in lung cancer cell lines compared with NL-20, particularly in A549 cells. Although these results require further experimental
\end{abstract}

Correspondence to: Professor Qiong Chen, Department of Gerontology, Xiangya Hospital of Central South University, 87 Xiangya Road, Changsha, Hunan 410078, P.R. China

E-mail: xj928114921@yeah.net

Key words: lung cancer, long non-coding RNAs, sex determining region Y-box 2 overlapping transcript, NCBP2 antisense RNA 2 verification, the analysis of 1ncRNA signatures between AC and SCC has provided insights into the regulatory mechanism of NSCLC development.

\section{Introduction}

Non-small-cell lung cancer (NSCLC) is the leading cause of cancer death worldwide (1). The most common subtypes of NSCLC are adenocarcinoma (AC) and squamous cell carcinoma (SCC) (2). Lung AC is the most common histological type of NSCLC, resulting in $>500,000$ deaths globally every year (3). In addition, SCC comprises $25-30 \%$ of pulmonary cancers (4). Despite advances in surgery, molecular subtyping and targeted therapy, prognosis of AC and NSCLC remains poor (5). However, the pathophysiological mechanisms contributing to AC and SCC remain largely unknown. Thus, there is an urgent need for the identification of novel agents that will provide clinicians with useful assistance in improving the survival rate of patients with AC and SCC.

Long non-coding RNAs (lncRNAs) are a large and important class of heterogeneous ncRNAs with a length $>200$ nucleotides $(6,7)$. LncRNAs are emerging contributors to the cancer paradigm, demonstrating potential roles in both oncogenic and tumor suppressive pathways (8). The human genome is replete with lncRNA, many of which are transcribed and likely to have a functional role. A number of dysregulated lncRNAs have been detected in multiple human cancers (9-11), and their expression is associated with cancer metastasis and prognosis. For example, the lncRNA Hox transcript antisense intergenic RNA (HOTAIR) is upregulated in primary breast tumors and metastases, and its overexpression is associated with enhanced breast cancer metastasis (12). Notably, lncRNA dysregulation was proposed as a hallmark feature in cancer (10). Recently, several lncRNA signatures were developed as novel predictors of survival in patients with cancer $(13,14)$, displaying a similar prognostic power to that of protein-coding RNA and microRNAs, thus providing a new molecular option for cancer diagnosis and prognosis. However, the survival rate of lncRNA signatures for lung cancer patients has not yet been investigated and the IncRNA-based pathophysiological mechanism contributing to AC and SCC have not been well characterized.

To investigate the underlying role of lncRNA in lung $\mathrm{AC}$ and SCC, the present study re-annotated a NSCLC 
microarray data analysis profiling to identify differentially expressed lncRNAs, then predicted the potential functions of these lncRNAs. Finally, some lncRNAs were validated using reverse transcription-quantitative polymerase chain reaction (RT-qPCR). A total of 65 lncRNAs were differentially expressed between AC and SCC. Of these lncRNAs, 3 lncRNAs were potentially related to cancer-associated pathways. Two of these lncRNAs were verified using RT-qPCR. Although these results require further experimental verification, the analysis of lncRNA signatures between AC and SCC provided interesting insights into the regulated mechanism of development of NSCLC.

\section{Materials and methods}

Cell culture and agents. The original microarray data were obtained from tumor tissues of $40 \mathrm{AC}$ and 18 SCC samples in NSCLC (https://www.ncbi.nlm.nih.gov/geo/query/acc .cgi?acc=GSE10245) (15). NSCLC cell lines A549, L78, H226, H520 and normal lung cell line NL-20 were obtained from Shanghai Institutes for Biological Sciences, Chinese Academy of Sciences (Shanghai, China). The cells were routinely cultured in RPMI-1640 (Invitrogen; Thermo Fisher Scientific, Inc., Waltham, MA, USA, Carlsbad USA) supplemented with $10 \%$ heat-inactivated fetal bovine serum (FBS; Invitrogen; Thermo Fisher Scientific, Inc.), $100 \mathrm{U} / \mathrm{ml}$ penicillin and $100 \mu \mathrm{g} / \mathrm{ml}$ streptomycin in a humidified cell incubator with an atmosphere of $5 \% \mathrm{CO}_{2}$ at $37^{\circ} \mathrm{C}$. Cells growing at an exponential rate were used for the experiments. Rabbit monoclonal glycogen synthase kinase-3 $\beta$ (GSK-3 $\beta$; ab32391), phosphorylated (p-)GSK-3 $\beta$ (ab131097), $\beta$-catenin (ab16051), $\mathrm{p}-\beta$-catenin (ab73153) and $\beta$-actin (ab8227), were obtained from Abcam (Cambridge, UK). Infrared (IR) fluorescent dye-labeled secondary antibody (A32731) was obtained from Thermo Fisher Scientific, Inc. Protease inhibitor mixture was obtained from Roche Diagnostics (Basel, Switzerland).

Microarray and Functional Reannotation. The microarray data set GSE10245 was downloaded from the Gene Expression Omnibus (GEO) database (http://www.ncbi.nlm .nih.gov/geo/query/acc.cgi?acc=GSE10245). The dataset was composed of the histological subtype AC samples $(n=40)$ and SCC samples ( $n=18)$ using Human Genome U133 (HG-U133) Plus 2.0 array (Affymetrix, Inc., Santa Clara, CA, USA).

Functional Reannotation of long non-coding RNAs. The probes on the HG-U133 Plus 2.0 array were reannotated for human lncRNA using non-coding RNA function annotation server (ncFANs) as described in its website (http://www .ebiomed.org/ncFANs/) (16). Differentially expressed lncRNAs were selected using the fold change method: lncRNAs with a fold change value $>2$ or $<0.5$ were considered as differentially expressed lncRNAs. In the present study, AC was considered as 'control'.

Obtaining co-expressing genes and functional enrichment for differentially expressed lncRNA. To explore potential functions of differentially expressed lncRNAs, co-expressing genes for each IncRNA were identified by calculating Pearson's correlation coefficient (PCC) between each differentially expressed lncRNA and all genes across all 18 samples. The genes with a strict cut-off of PCC $>0.75$ or $<-0.75$ ) were identified as co-expressing genes. Pathway enrichment was then implemented to identify the affected pathways of IncRNA co-expressing genes by using the Database for Annotation, Visualization and Integrated Discovery (DAVID) version $6.7(17,18)$.

$R T-q P C R$. Total RNA was extracted using TRIzol (Invitrogen; Thermo Fisher Scientific, Inc.) according to the manufacturer's protocol and diluted to $200 \mathrm{ng} / \mathrm{ml}$. RT-qPCR was then performed using One Step SYBR ${ }^{\circledR}$ Prime Script ${ }^{\text {TM }}$ RT-PCR Kit II (Takara Biotechnology Co., Ltd., Dalian, China) according to the manufacturer's protocol. To be brief, two stages were included: Stage I for reverse transcription, $42^{\circ} \mathrm{C} 5 \mathrm{~min}, 95^{\circ} \mathrm{C}$ $10 \mathrm{sec}$; stage II for qPCR reaction, denaturation: $95^{\circ} \mathrm{C} 5 \mathrm{sec}$, annealing and extension: $60^{\circ} \mathrm{C} 34 \mathrm{sec}$, repeated 35 cycles. All data were collected from three independent experiments. GAPDH was used as an internal control and relative gene expression was calculated using the $2^{-\Delta \Delta \mathrm{Cq}}$ method (19). Primers used in RT-qPCR were as follows: Sex determining region Y-box 2 overlapping transcript (SOX2-OT), forward 5'-CGAAATGGATTCACGGTGCC-3' and reverse 5'-TGC CAGATCAGGGTGTTGTC-3'; NCBP2 antisense RNA 2 (NCBP2-AS2), forward 5'-TTTAATCCTGGGCTGTGC GG-3' and reverse 5'-CACAAGACTTCAACGGAGCG-3'; and GAPDH, forward 5'-GGACCAATACGACCAAAT CCG-3' and reverse 5'-AGCCACATCGCTCAGACAC-3'.

Protein extraction. Whole cell proteins were extracted using RIPA buffer (P0013C; Beyotime Institute of Biotechnology, Shanghai, China) according to the manufacturer's protocol. In brief, cells were washed with PBS and scraped with RIPA buffer. Cells were collected in microfuge tubes and agitated for $30 \mathrm{~min}$ at $4^{\circ} \mathrm{C}$. Then, centrifugation was performed at $16,000 \mathrm{x} \mathrm{g}$ for $20 \mathrm{~min}$ at $4^{\circ} \mathrm{C}$. The supernatants were collected in a fresh tube for western blot analysis. Protein concentration was measured using Bradford assay (P0006; Beyotime Institute of Biotechnology).

Western blot analysis. A total of $50 \mu \mathrm{g}$ proteins from the cells were separated by $10-12 \%$ SDS-PAGE and transferred to nitrocellulose membrane (Pall Life Sciences, Port Washington, NY, USA). Membranes were blocked with 5\% milk at room temperature for $30 \mathrm{~min}$, then incubated overnight at $4^{\circ} \mathrm{C}$ with $\beta$-catenin (1:1,000 dilution), $\mathrm{p}$ - $\beta$-catenin (1:1,000 dilution), $\mathrm{p}-\mathrm{GSK}-3 \beta$ (1:500 dilution), GSK-3 $\beta$ (1:500 dilution) or $\beta$-actin (1:1,000 dilution) antibodies. Infrared (IR) fluorescent dye-labeled secondary antibody $(1: 10,000)$ was incubated with the membrane at room temperature for $1 \mathrm{~h}$. Western blot bands were visualized using an IR Imaging System (LI-COR Biosciences, Lincoln, NE, USA). Band densities for each group were quantified using Odyssey software version 3.0 (LI-COR Biosciences) and normalized to $\beta$-actin expression.

Statistical analysis. Data were obtained from 3-6 independent experiments and are presented as the mean \pm standard deviation. Data were evaluated by analysis of variance followed by Student-Newman-Keuls post hoc tests using the SPSS 


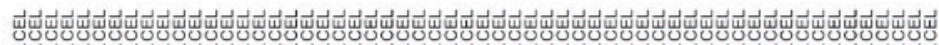

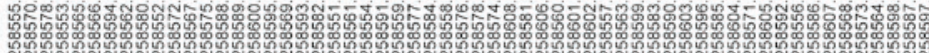

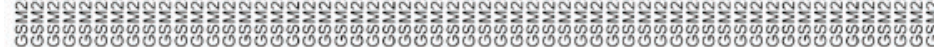
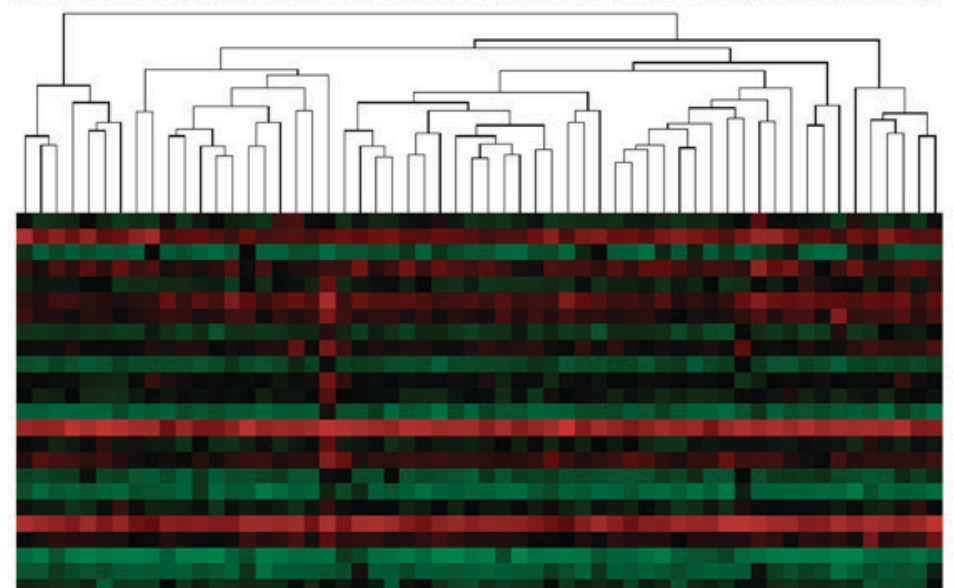

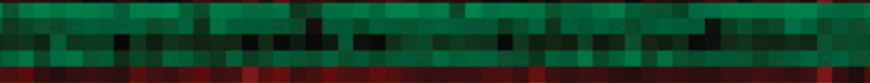
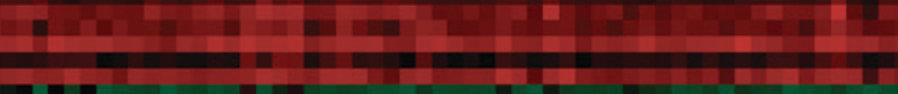

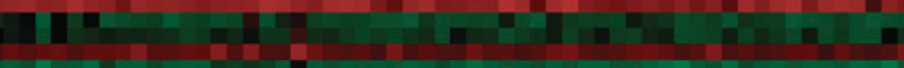

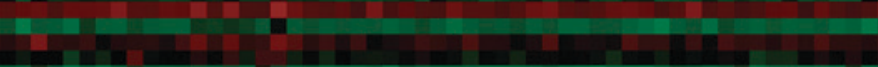

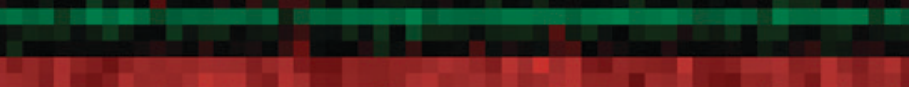

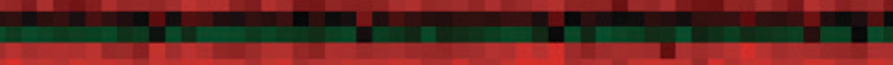

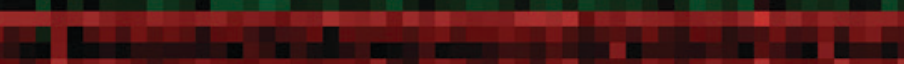

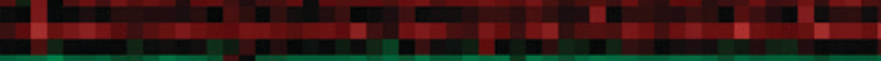

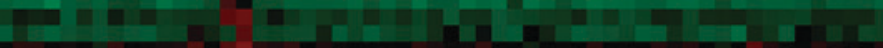

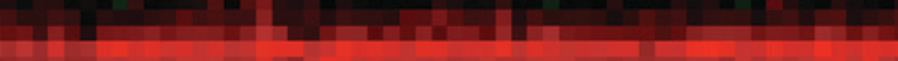

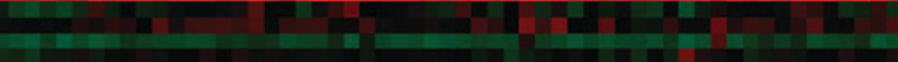

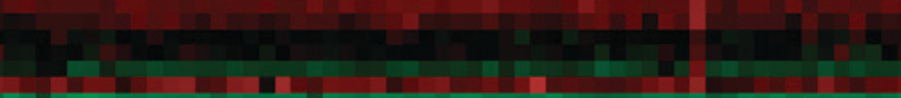

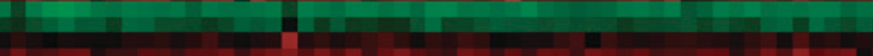

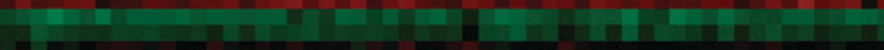

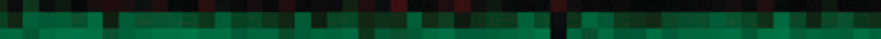

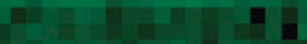
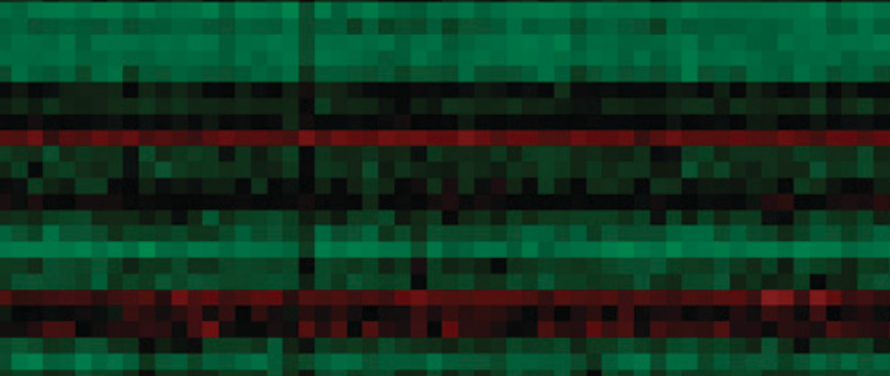

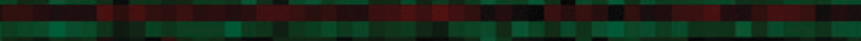

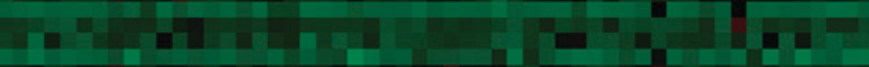

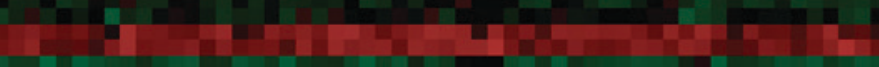

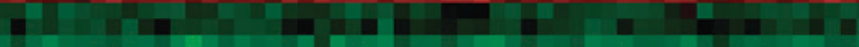

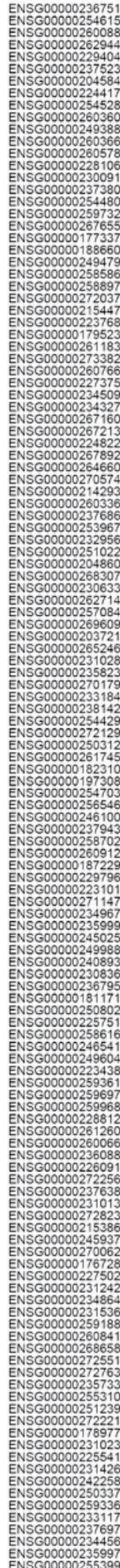

Figure 1. Bidirectional hierarchical cluster of long non-coding RNA profiles. 
Table I. Differentially expressed lncRNAs.

\begin{tabular}{|c|c|}
\hline IncRNA & $\log$ (fold change) \\
\hline ENSG00000253898 & -4.152017003 \\
\hline ENSG00000235123 & -3.540259841 \\
\hline ENSG00000262117 & -2.746729646 \\
\hline ENSG00000245750 & -2.61388764 \\
\hline ENSG00000272620 & -2.564460843 \\
\hline ENSG00000225383 & -2.358113042 \\
\hline ENSG00000260943 & -2.270111418 \\
\hline ENSG00000264868 & -2.21214258 \\
\hline ENSG00000266968 & -2.202088651 \\
\hline ENSG00000204832 & -1.931326052 \\
\hline ENSG00000256969 & -1.767283812 \\
\hline ENSG00000259974 & -1.678282345 \\
\hline ENSG00000235584 & -1.633014477 \\
\hline ENSG00000260997 & -1.563708541 \\
\hline ENSG00000240476 & -1.555238143 \\
\hline ENSG00000232018 & -1.515156717 \\
\hline ENSG00000223414 & -1.454123693 \\
\hline ENSG00000233834 & -1.366598222 \\
\hline ENSG00000237499 & -1.278009264 \\
\hline ENSG00000250328 & -1.221423553 \\
\hline ENSG00000251629 & -1.205091444 \\
\hline ENSG00000230487 & -1.177625469 \\
\hline ENSG00000247134 & -1.155801947 \\
\hline ENSG00000254429 & -1.074388165 \\
\hline ENSG00000241295 & -1.053131154 \\
\hline ENSG00000272732 & -1.042988456 \\
\hline ENSG00000253563 & -1.015484492 \\
\hline ENSG00000214049 & -1.000246779 \\
\hline ENSG00000266401 & 1.003846483 \\
\hline ENSG00000246859 & 1.005233819 \\
\hline ENSG00000231971 & 1.021838364 \\
\hline ENSG00000215808 & 1.034891987 \\
\hline ENSG00000034063 & 1.042316963 \\
\hline ENSG00000267034 & 1.093965748 \\
\hline ENSG00000245526 & 1.106449907 \\
\hline ENSG00000225484 & 1.124075352 \\
\hline ENSG00000233864 & 1.136818147 \\
\hline ENSG00000255135 & 1.169779461 \\
\hline ENSG00000270170 & 1.195208999 \\
\hline ENSG00000261668 & 1.208732331 \\
\hline ENSG00000271856 & 1.217547617 \\
\hline ENSG00000226363 & 1.245074712 \\
\hline ENSG00000273190 & 1.26525531 \\
\hline ENSG00000234715 & 1.278314773 \\
\hline ENSG00000227640 & 1.304396623 \\
\hline ENSG00000228630 & 1.32037165 \\
\hline ENSG00000261409 & 1.373814297 \\
\hline ENSG00000254560 & 1.389245877 \\
\hline ENSG00000110347 & 1.461512858 \\
\hline ENSG00000261521 & 1.470414154 \\
\hline ENSG00000269994 & 1.499538599 \\
\hline ENSG00000230937 & 1.584953003 \\
\hline
\end{tabular}

Table I. Continued.

\begin{tabular}{ll}
\hline lncRNA & $\log$ (fold change) \\
\hline ENSG00000225826 & 1.638461788 \\
ENSG00000213963 & 1.851214762 \\
ENSG00000249082 & 1.942238906 \\
ENSG00000224259 & 1.961743949 \\
ENSG00000233901 & 1.962040139 \\
ENSG00000241732 & 1.968491957 \\
ENSG00000104725 & 2.438796861 \\
ENSG00000261116 & 2.754710633 \\
ENSG00000245648 & 2.758743567 \\
ENSG00000260581 & 2.93322784 \\
ENSG00000265992 & 3.470736871 \\
ENSG00000242512 & 3.647947373 \\
ENSG00000242808 & 5.237044135 \\
\hline lncRNA, long non-coding RNA. & \\
\hline
\end{tabular}

software v.19.0 (IBM Corp., Armonk, NY, USA) and illustrated using GraphPad Prism version 5.0 (GraphPad Software, Inc., La Jolla, CA, USA). P $<0.05$ was considered to indicate a statistically significant difference.

\section{Results and Discussion}

Differentially expressed lncRNAs between AC and SCC subtypes. Following functional annotation using ncFANs and calculating fold change value, mRNA and lncRNA profiles were obtained. As a result of the bidirectional hierarchical cluster of IncRNA profiles, it was observed that the samples were clustered into classes approximately corresponding to AC and SCC, suggesting that the re-annotated profile could be used for subsequent analysis (Fig. 1).

Following analysis, a total of 65 lncRNAs were considered differentially expressed (Table I). Of these $65 \mathrm{lncRNA}$, 28 were downregulated in the SCC subtypes compared with AC subtypes. By contrast, 37 lncRNAs were upregulated in SCC compared with AC. The most upregulated lncRNA was SOX2-OT (ENSG00000242808; Table I). SOX2-OT is located on human chromosome $3 \mathrm{q} 26.33$, which is frequently amplified in lung SCCs (20). Recent studies have demonstrated a positive role for it in transcriptional regulation of SOX2 $(21,22)$. Similarly to SOX2, SOX2OT is highly expressed in embryonic stem cells and downregulated upon induction of differentiation (23). SOX2OT is dynamically regulated during the embryogenesis of vertebrates, and delimited to the brain in adult mice and human (23). Shahryari et al (23) investigated the role of SOX2OT in lung cancer and found that the expression level of SOX2-OT is significantly higher in SCCs than in ACs of the lung, which is in agreement with a previous study by this group (20). Another study indicated that SOX2-OT is a novel prognostic factor (24). In brief, SOX2-OT is important in regulating lung cancer cell proliferation and may be a potential novel prognostic indicator $(20,23,25)$. 


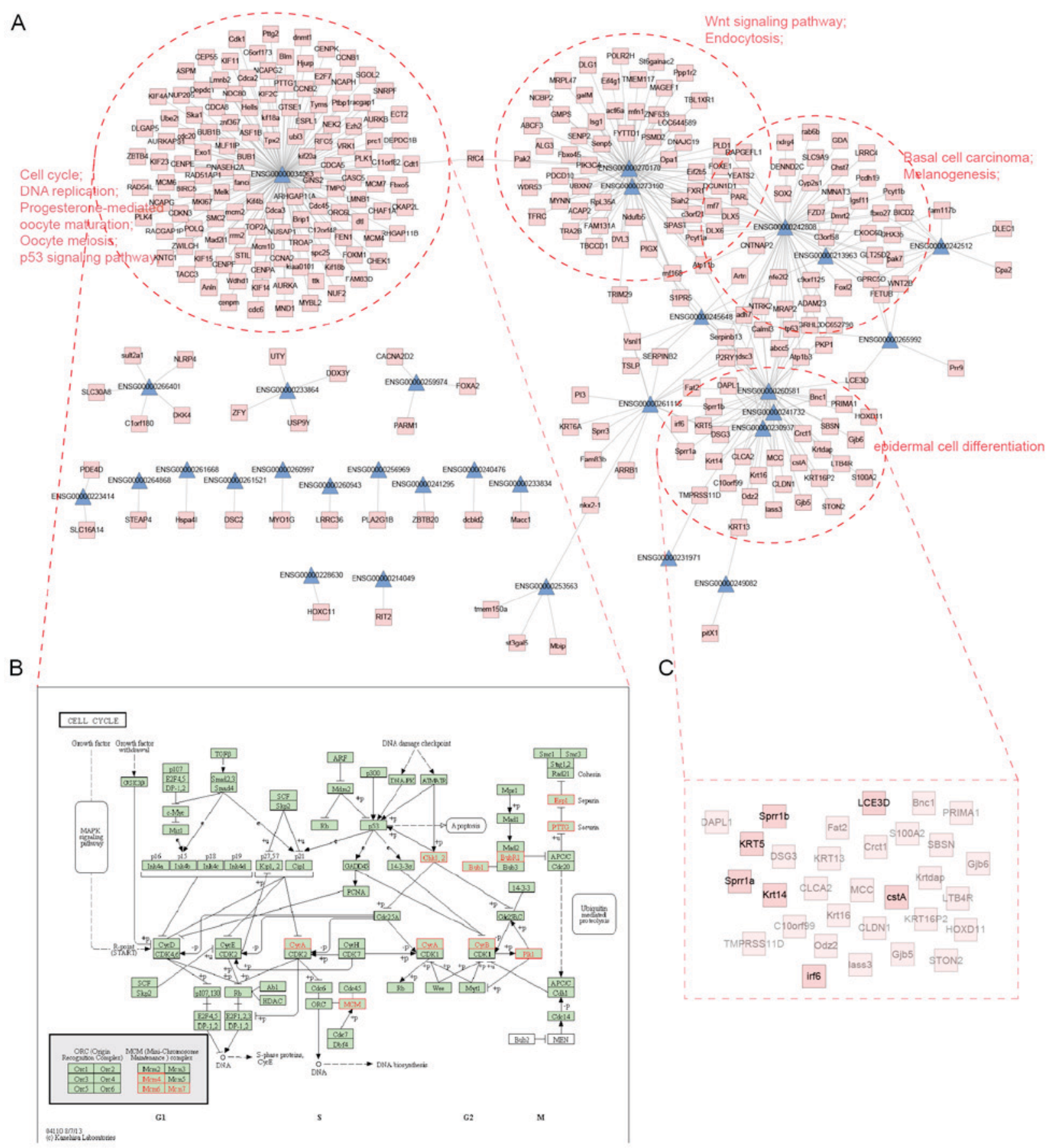

Figure 2. (A) The co-expressing network of differentially expressed lncRNAs and mRNAs. (B) Cell cycle pathway annotated with the co-expressing genes of IncRNA-UHRF1. (C) The co-expressing genes of three lncRNAs. IncRNA, long non-coding RNA.

The most downregulated IncRNA was LINC01419 (ENSG00000253898; Table I). Although, to the best of our knowledge, there have been no reports about this lncRNA in lung cancer, Zhang et al (26) validated that LINC01419 was significantly overexpressed in hepatitis B- and C-related $\mathrm{HCC}$ when compared with matched non-tumor liver tissues. Furthermore, functional predictions suggested that LINC01419 regulates cell cycle genes (26).

The method of ncFANs has been applied to different cancer types and other diseases. For example, in the studies of Gao et al $(27,28)$, using the functional annotation algorithm from ncFANs, some differentially expressed lncRNAs were identified in tongue SCC tissues (e.g. SPRR2D-1) and in nasopharyngeal carcinoma (e.g. C22orf32-1). These lncRNAs were both different from the results of the present study, indicating that this is not a common phenomenon or specific to a certain cancer type.

LncRNA-gene co-expression network and functional enrichment. Following calculation of the PCC between differentially expressed lncRNAs and all genes, significantly co-expressed genes were identified for these lncRNAs. A lncRNA-gene co-expression network was then constructed, in which nodes were lncRNAs and genes, and were connected if they are co-expressed (Fig. 2A); in this network, there were 31 lncRNA nodes. The potential biological functions of these differentially expressed lncRNAs were then explored using DAVID v.6.7 
Table II. Enriched pathways of corresponding co-expressed genes of differentially expressed long non-coding RNAs.

\begin{tabular}{lllll}
\hline difLnc & Pathway-ID & \multicolumn{1}{c}{ Pathway name } & P-value & \multicolumn{1}{c}{ Gene } \\
\hline ENSG00000034063 & Path:04110 & Cell cycle & $2.82 \times 10^{-14}$ & $1111 ; 4173 ; 4175 ; 4176 ; 5347 ;$ \\
& & & & $699 ; 701 ; 890 ; 891 ; 9133 ; 9232 ; 9700$ \\
ENSG00000034063 & Path:03030 & DNA replication & $3.09 \times 10^{-9}$ & $10535 ; 2237 ; 4173 ; 4175 ; 4176 ; 5985$ \\
ENSG00000034063 & Path:04914 & $\begin{array}{l}\text { Progesterone-mediated } \\
\text { oocyte maturation }\end{array}$ & $1.60 \times 10^{-5}$ & $5347 ; 699 ; 890 ; 891 ; 9133$ \\
ENSG00000034063 & Path:04114 & Oocyte meiosis & $4.91 \times 10^{-5}$ & $5347 ; 6790 ; 699 ; 9232 ; 9700$ \\
ENSG00000034063 & Path:04115 & p53 signaling pathway & $9.80 \times 10^{-5}$ & $1111 ; 51512 ; 891 ; 9133$ \\
ENSG00000242808 & Path:05217 & Basal cell carcinoma & $2.95 \times 10^{-3}$ & $7482 ; 8324$ \\
ENSG00000242808 & Path:04916 & Melanogenesis & $9.45 \times 10^{-3}$ & $7482 ; 8324$ \\
ENSG00000270170 & Path:04310 & Wnt signaling pathway & $2.44 \times 10^{-3}$ & $1857 ; 59343 ; 79718$ \\
ENSG00000270170 & Path:04144 & Endocytosis & $5.69 \times 10^{-3}$ & $23527 ; 5337 ; 7037$ \\
\hline
\end{tabular}

difLnc, differentially expressed long non-coding RNAs.

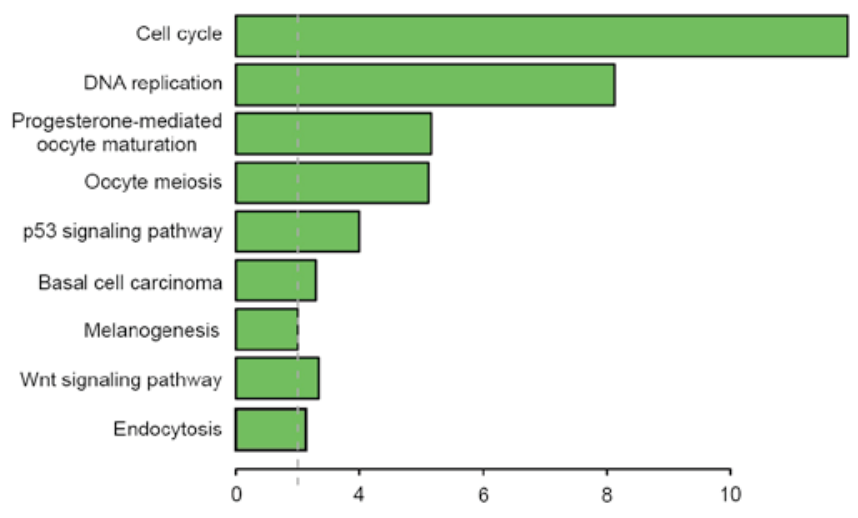

Figure 3. The enriched pathways of differentially expressed lncRNAs. The dashed line indicates the cutoff value.

for corresponding co-expressed genes, which established that there was enrichment in some cancer-related biological processes, including the cell cycle, p53 signaling pathway and Wnt signaling pathway (Figs. 2A and 3; Table II). To better illustrate the regulated roles of lncRNAs, the co-expressed genes of lncRNA-UHRF1 were annotated into pathways of the cell cycle (Fig. 2B); potential regulation of some key proteins in this pathway was identified, including minichromosome maintenance complex component 2 (MCM2), cyclin A2 (CCNA2, also known as $\mathrm{CycA}$ ), cyclin B1 (CCNB1, also known as $\mathrm{CycB}$ ), checkpoint kinase 1 (CHEK1, also known as Chk1,2) and checkpoint kinase 2 (CHEK2, also known as Chk2). Zhang et al (29) investigated whether lovastatin inhibits proliferation due to MCM2 in NSCLCs. Using RT-qPCR and western blot analysis, they demonstrated that lovastatin treatment markedly and consistently inhibits the expression of MCM2. MCM2 knockdown inhibited expression of retinoblastoma protein, cyclin D1 and cyclin dependent kinase 4, but increased expression of cyclin dependent kinase inhibitor $1 \mathrm{~A}$ (p21) and tumor protein p53, suggesting that siMCM2 triggered cell cycle arrest (29).

CCNA2, a secreted matrix-associated molecule, is involved in multiple cellular processes (30). Previous studies have indicated that expression of CCNA2 is inversely correlated with the aggressiveness of NSCLC (31). Jim et al (32) used 3 NSCLC cell line systems to demonstrate that long-term treatment of cells with recombinant CCNA2 leads to a permanent cell cycle arrest in G1 phase, in addition to apoptosis assays to demonstrate that cells remain viable. Furthermore, CCNA2-treated NSCLCs acquire phenotypic characteristics of senescent cells, including an enlarged and flattened cell shape and expression of senescence-associated $\beta$-galactosidase (32).

ATR serine/threonine kinase, a DNA damage response kinase, and its effector kinase, CHEK1, are required for cancer cells to survive oncogene-induced replication stress (33). High expression of CHEK1 in lung tumors is associated with poor overall survival (34); CHEK1 has previously been demonstrated to be a direct target of microRNA-195, which decreases CHEK1 expression in lung cancer cells.

Notably, the present study demonstrated that 3 differentially expressed 1ncRNAs, ENSG00000241732, ENSG00000260581 and ENSG00000230937, co-regulated some mRNAs: The co-expressed genes of these lncRNAs are presented in solid boxes in Fig. 2C. Of these genes, late cornified envelope 3D (LCE3D) has previously been identified as significantly upregulated in head and neck cancer cells compared with normal mucosa (35). Wright et al (36) investigated the possible role of interferon regulatory factor 6 (IRF6) in asbestos lung tumorigenicity, its potential utility as a marker of asbestos related lung cancer for purposes of causal attribution and its potential as a treatment target for lung cancers arising in asbestos exposed persons. Overexpression of small proline rich protein 1B (SPRR1B) in bronchial epithelial cells is a marker for early metaplastic changes induced by various toxicants/carcinogens (37). Numerous studies have established the role of SPRR1B during squamous differentiation of skin and respiratory epithelial cells (38). Woenckhaus et al (39) investigated the gene expression in surgically resected and microdissected samples of NSCLCs, matched normal bronchial epithelium, and peripheral lung tissue from both smokers and non-smokers using an Affymetrix U133A array: They 
A

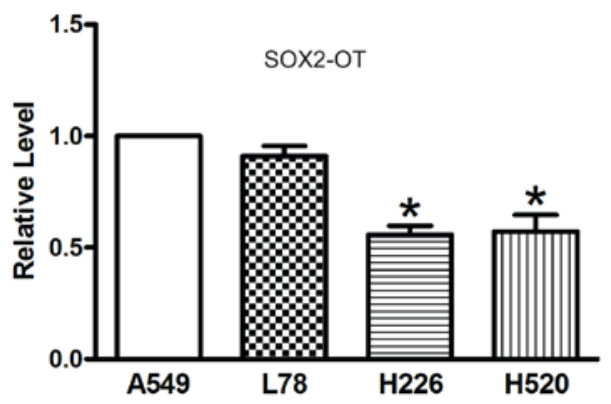

B

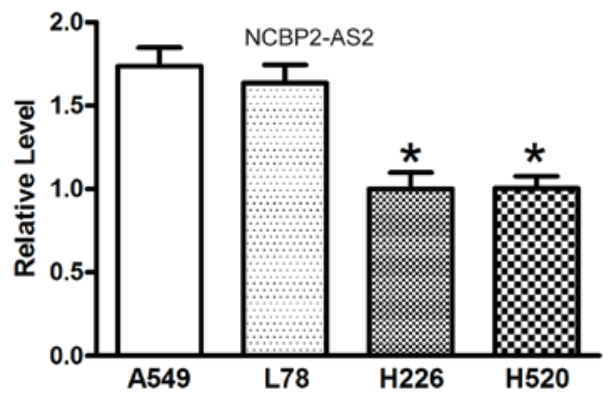

Figure 4. Expression of long non-coding RNAs (A) SOX2-OT and (B) NCBP2-AS2 in A549, L78, H226 and H520 cells, respectively. Data are expressed as the mean \pm standard error of the mean $(n=3)$. "P<0.05 vs. normal group. SOX2-OT, sex determining region Y-box 2 overlapping transcript; NCBP2-AS2, NCBP2 antisense RNA 2.

A
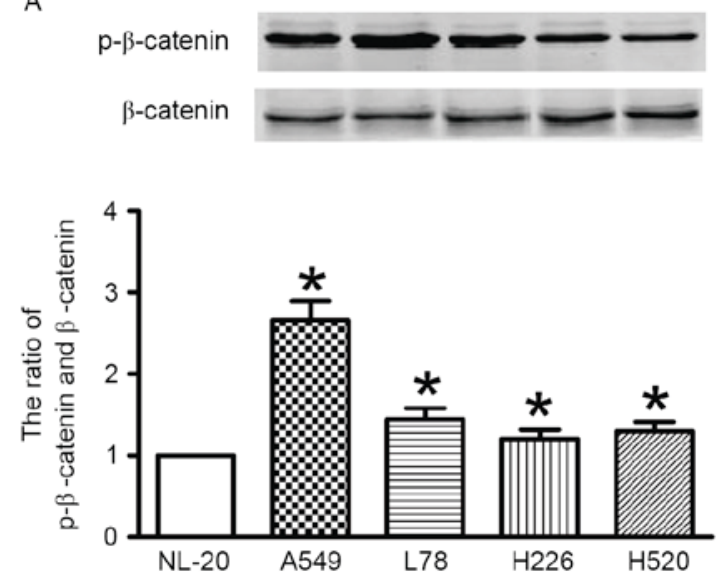

B
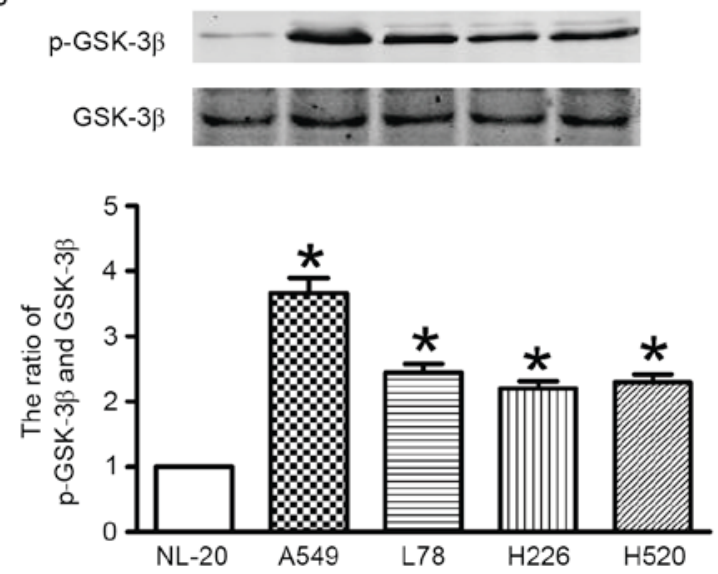

Figure 5. Activation of Wnt signaling pathway proteins in normal and lung cancer cells following transfection with long non-coding RNAs. (A) Phosphorylation of $\beta$-catenin following transfection with SOX2-OT. (B) Phosphorylation of GSK-3 $\beta$ following transfection with NCBP2-AS2. Data are expressed as the mean \pm standard error of the mean $(n=3)$. "P<0.05 vs. normal group. $\mathrm{p}$-, phosphorylated; SOX2-OT, sex determining region Y-box 2 overlapping transcript; GSK-3 $\beta$, glycogen synthase kinase $3 \beta ;$ NCBP2-AS2, NCBP2 antisense RNA 2.

demonstrated that SPRR1B is differentially expressed and is related to cell differentiation (39).

Expression of IncRNA1 and LncRNA2 in AC and SCC cells. Finally, 2 lncRNAs were selected for further investigation: SOX2-OT and NCBP2-AS2. These IncRNAs were selected since SOX2-OT was the most upregulated lncRNA and NCBP2-AS2 potentially regulates the cancer-associated Wnt signaling pathway (Table II) (40). To explore SOX2-OT and NCBP2-AS2 expression in NSCLC cells, RT-qPCR was used to determine SOX2-OT levels in normal lung cell line NL-20 and cancer cell lines A549, L78, H226 and H520. As demonstrated in Fig. 4A, SOX2-OT was significantly upregulated in AC cells (A549 and L78) compared with the SCC cells (H226 and H520). NCBP2-AS2 levels were also significantly upregulated in AC cells compared with the SCC cells $(\mathrm{P}<0.05$; Fig. 4B). In addition, levels of SOX2-OT and NCBP2-AS2 were both significantly lower in NL-20 cell lines compared with AC and SCC cells (Fig. 4A and B).

Expression levels of Wht signaling pathway proteins following treatment with lncRNA1 and lncRNA2 in AC and SCC cells. Deregulation of the Wnt signaling pathway is important in the initiation and development of lung cancers. The present study examined whether the Wnt signaling pathway was involved in different lung cancer cells lines. Western blot assays demonstrated that the expression of $\beta$-catenin and GSK-3 $\beta$ was not altered in cancer cell lines compared with NL-20, but increased p- $\beta$-catenin and p-GSK- $3 \beta$ was detected in lung cancer cell lines compared with NL-20, particularly A549 cells (Fig. 5). These results demonstrated the potentially different involvement of the Wnt signaling pathway in the two types of NSCLC cancer (AC and SCC).

In conclusion, in the present study, differentially expressed lncRNAs between lung AC and SCC were identified by re-annotation of NSCLC microarray data analysis profiling. A total of 65 lncRNAs were differentially expressed between AC and SCC. The potential functions of these lncRNAs were then predicted. Finally, 2 of these lncRNAs were investigated using RT-qPCR. Although these results require further experimental verification, the analysis of lncRNA signatures between AC and SCC still provided insights into the regulated mechanism of development of NSCLC.

\section{References}

1. Croegaert K and Kolesar JM: Role of anaplastic lymphoma kinase inhibition in the treatment of non-small-cell lung cancer. Am J Health Syst Pharm 72: 1456-1462, 2015. 
2. Meng G, Wang W, Chai K, Yang S, Li F and Jiang K: Combination treatment with triptolide and hydroxycamptothecin synergistically enhances apoptosis in A549 lung adenocarcinoma cells through PP2A-regulated ERK, p38 MAPKs and Akt signaling pathways. Int J Oncol 46: 1007-1017, 2015.

3. Imielinski M, Berger AH, Hammerman PS, Hernandez B, Pugh TJ, Hodis E, Cho J, Suh J, Capelletti M, Sivachenko A, et al: Mapping the hallmarks of lung adenocarcinoma with massively parallel sequencing. Cell 150: 1107-1120, 2012.

4. Zhu Y, Xing $P$ and Li J: Treatment of advanced squamous cell lung cancer. Zhongguo Fei Ai Za Zhi 19: 687-691, 2016 (In Chinese).

5. Di JZ, Peng JY and Wang ZG: Prevalence, clinicopathological characteristics, treatment, and prognosis of intestinal metastasis of primary lung cancer: A comprehensive review. Surg Oncol 23 : 72-80, 2014

6. Kapranov P, Cheng J, Dike S, Nix DA, Duttagupta R, Willingham AT, Stadler PF, Hertel J, Hackermüller J, Hofacker IL, et al: RNA maps reveal new RNA classes and a possible function for pervasive transcription. Science 316 : 1484-1488, 2007

7. Chen X: KATZLDA: KATZ measure for the lncRNA-disease association prediction. Sci Rep 5: 16840, 2015.

8. Gibb EA, Brown CJ and Lam WL: The functional role of long non-coding RNA in human carcinomas. Mol Cancer 10: 38, 2011.

9. Qiu MT, Hu JW, Yin R and Xu L: Long noncoding RNA: An emerging paradigm of cancer research. Tumour Biol 34: 613-620, 2013.

10. Gutschner T and Diederichs S: The hallmarks of cancer: A long non-coding RNA point of view. RNA Biol 9: 703-719, 2012.

11. Tsai MC, Spitale RC and Chang HY: Long intergenic noncoding RNAs: New links in cancer progression. Cancer Res 71: 3-7, 2011

12. Gupta RA, Shah N, Wang KC, Kim J, Horlings HM, Wong DJ, Tsai MC, Hung T, Argani P, Rinn JL, et al: Long non-coding RNA HOTAIR reprograms chromatin state to promote cancer metastasis. Nature 464: 1071-1076, 2010.

13. Zhang XQ, Sun S, Lam KF, Kiang KM, Pu JK, Ho AS, Lui WM, Fung CF, Wong TS and Leung GK: A long non-coding RNA signature in glioblastoma multiforme predicts survival. Neurobiol Dis 58: 123-131, 2013.

14. Meng J, Li P, Zhang Q, Yang Z and Fu S: A four-long non-coding RNA signature in predicting breast cancer survival. J Exp Clin Cancer Res 33: 84, 2014.

15. Kuner R, Muley T, Meister M, Ruschhaupt M, Buness A, Xu EC Schnabel P, Warth A, Poustka A, Sültmann H and Hoffmann H: Global gene expression analysis reveals specific patterns of cell junctions in non-small cell lung cancer subtypes. Lung Cancer 63: 32-38, 2009.

16. Liao Q, Xiao H, Bu D, Xie C, Miao R, Luo H, Zhao G, Yu K, Zhao H, Skogerb $\varnothing$, et al: ncFANs: A web server for functional annotation of long non-coding RNAs. Nucleic Acids Res 39 (Web Server issue): W118-W124, 2011

17. Huang da W, Sherman BT and Lempicki RA: Systematic and integrative analysis of large gene lists using DAVID bioinformatics resources. Nat Protoc 4: 44-57, 2009.

18. Huang da W, Sherman BT and Lempicki RA: Bioinformatics enrichment tools: Paths toward the comprehensive functional analysis of large gene lists. Nucleic Acids Res 37: 1-13, 2009.

19. Livak KJ and Schmittgen TD: Analysis of relative gene expression data using real-time quantitative PCR and the 2(-Delta Delta C(T)) method. Methods 25: 402-408, 2001

20. Hou Z, Zhao W, Zhou J, Shen L, Zhan P, Xu C, Chang C, Bi H, Zou J, Yao X, et al: A long noncoding RNA Sox2ot regulates lung cancer cell proliferation and is a prognostic indicator of poor survival. Int J Biochem Cell Biol 53: 380-388, 2014.

21. Shahryari A, Rafiee MR, Fouani Y, Oliae NA, Samaei NM, Shafiee M, Semnani S, Vasei M and Mowla SJ: Two novel splice variants of SOX2OT, SOX2OT-S1, and SOX2OT-S2 are coupregulated with SOX2 and OCT4 in esophageal squamous cell carcinoma. Stem Cells 32: 126-134, 2014.

22. Askarian-Amiri ME, Seyfoddin V, Smart CE, Wang J, Kim JE, Hansji H, Baguley BC, Finlay GJ and Leung EY: Emerging role of long non-coding RNA SOX2OT in SOX2 regulation in breast cancer. PLoS One 9: e102140, 2014.
23. Shahryari A, Jazi MS, Samaei NM and Mowla SJ: Long non-coding RNA SOX2OT: Expression signature, splicing patterns, and emerging roles in pluripotency and tumorigenesis. Front Genet 6: 196, 2015.

24. Zhang Y, Yang R, Lian J and Xu H: LncRNA Sox2ot overexpression serves as a poor prognostic biomarker in gastric cancer. Am J Transl Res 8: 5035-5043, 2016.

25. Saghaeian Jazi M, Samaei NM, Ghanei M, Shadmehr MB and Mowla SJ: Identification of new SOX2OT transcript variants highly expressed in human cancer cell lines and down regulated in stem cell differentiation. Mol Biol Rep 43: 65-72, 2016.

26. Zhang H, Zhu C, Zhao Y, Li M, Wu L, Yang X, Wan X, Wang A, Zhang MQ, Sang $X$ and Zhao H: Long non-coding RNA expression profiles of hepatitis $\mathrm{C}$ virus-related dysplasia and hepatocellular carcinoma. Oncotarget 6: 43770-43778, 2015.

27. Gao W, Chan JY and Wong TS: Long non-coding RNA deregulation in tongue squamous cell carcinoma. Biomed Res Int 2014: 405860, 2014.

28. Gao W, Chan JY and Wong TS: Differential expression of long noncoding RNA in primary and recurrent nasopharyngeal carcinoma. Biomed Res Int 2014: 404567, 2014.

29. Zhang X, Teng Y, Yang F, Wang M, Hong X, Ye LG, Gao YN and Chen GY: MCM2 is a therapeutic target of lovastatin in human non-small cell lung carcinomas. Oncol Rep 33: 2599-2605, 2015.

30. Jandova J, Beyer TE, Meuillet EJ and Watts GS: The matrix protein CCN1/CYR61 is required for a(V)b5-mediated cancer cell migration. Cell Biochem Funct 30: 687-695, 2012.

31. Habel N, Vilalta M, Bawa O, Opolon P, Blanco J and Fromigué O: Cyr61 silencing reduces vascularization and dissemination of osteosarcoma tumors. Oncogene 34: 3207-3213, 2015.

32. Jim Leu SJ, Sung JS, Chen MY, Chen CW, Cheng JY, Wang TY and Wang JJ: The matricellular protein $\mathrm{CCN} 1$ suppresses lung cancer cell growth by inducing senescence via the p53/p21 pathway. J Cell Biochem 114: 2082-2093, 2013.

33. Mohni KN, Kavanaugh GM and Cortez D: ATR pathway inhibition is synthetically lethal in cancer cells with ERCC1 deficiency. Cancer Res 74: 2835-2845, 2014.

34. Liu B, Qu J, Xu F, Guo Y, Wang Y, Yu H and Qian B: MiR-195 suppresses non-small cell lung cancer by targeting CHEK1. Oncotarget 6: 9445-9456, 2015.

35. Tyszkiewicz T, Jarzab M, Szymczyk C, Kowal M, Krajewska J, Jaworska M, Fraczek M, Krajewska A, Hadas E, Swierniak M, et al: Epidermal differentiation complex (locus 1q21) gene expression in head and neck cancer and normal mucosa. Folia Histochem Cytobiol 52: 79-89, 2014.

36. Wright CM, Larsen JE, Hayward NK, Martins MU, Tan ME, Davidson MR, Savarimuthu SM, McLachlan RE, Passmore LH, Windsor MN, et al: ADAM28: A potential oncogene involved in asbestos-related lung adenocarcinomas. Genes Chromosomes Cancer 49: 688-698, 2010.

37. Reddy SP, Vuong H and Adiseshaiah P: Interplay between proximal and distal promoter elements is required for squamous differentiation marker induction in the bronchial epithelium: Role for ESE-1, Sp1 and AP-1 proteins. J Biol Chem 278: 21378-21387, 2003.

38. Tesfaigzi Y, Wright PS and Belinsky SA: SPRR1B overexpression enhances entry of cells into the G0 phase of the cell cycle. Am J Physiol Lung Cell Mol Physiol 285: L889-L898, 2003.

39. Woenckhaus M, Klein-Hitpass L, Grepmeier U, Merk J, Pfeifer M, Wild P, Bettstetter M, Wuensch P, Blaszyk H, Hartmann A, et al: Smoking and cancer-related gene expression in bronchial epithelium and non-small-cell lung cancers. J Pathol 210: 192-204, 2006.

40. Li J, Huang KL, Zhang T, Li H, Zhao J and Wang H: Pan-cancer methylation and expression profiling of adenocarcinomas revealed epigenetic silencing in the WNT signaling pathway. Neoplasma 63: 208-214, 2016. 